\title{
Examining the influence of illness perception and financial toxicity on the quality of life of prostate cancer patients
}

\author{
Elizabeth O. Akin-Odanye ${ }^{1,2^{*}}$ (D), Chidiebere N. Ogo ${ }^{2,3}$, Faoziyat A. Sulaiman ${ }^{2,4}$, Lawal Suleiman ${ }^{5,2}$, \\ Motolani E. Ogunsanya ${ }^{2,6}$ and Folakemi T. Odedina ${ }^{2,7}$
}

\begin{abstract}
Background: Cancer of the prostate $(\mathrm{CaP})$ is a public health problem that affects the male genitourinary system causing a significant threat to men's quality of life (QoL). Experiencing financial constraints and poor illness perception may further compromise the QoL of men with CaP.

Methods: Aim: To examine the relationship between financial toxicity and illness perception with quality of life in men with CaP. The descriptive cross-sectional study used simple random sampling technique to recruit 173 men with CaP from four tertiary health facilities in Nigeria. Data were collected with the comprehensive score for financial toxicity (COST-FACIT), the brief illness perception questionnaire (Brief IPQ) and the functional assessment of cancer therapy-prostate (FACT-P). Analysis of data was carried out using analysis of variance, correlation and hierarchical regression analyses.
\end{abstract}

Results: The 173 participants had an average age of $71.57 \pm 11.18$, and $53.18 \%$ had one comorbid disease. Significant difference was found in overall QoL based on treatment site and number of comorbid diseases $(P<0.01)$. QoL had a significant inverse relationship with all the illness perception variables and a significant linear relationship with lower financial toxicity $(P<0.01)$. Furthermore, financial toxicity $(P<0.05)$ and four illness perception variables: consequences, identity, concern and illness understanding $(P<0.01)$, had significant individual influences on QoL of men with CaP.

Conclusions: Quality of life in men with CaP may be improved through mitigating the financial toxicity associated with accessing care and providing appropriate counseling about the illness and what to expect following prostate cancer diagnosis and during treatment.

Keywords: Prostate cancer, Illness perceptions, Financial toxicity, Quality of life

\section{Background}

Cancer of the prostate $(\mathrm{CaP})$ is one of the most commonly diagnosed male malignancies globally [1]. It is a public health problem that affects the male genitourinary system causing a significant threat to their health and quality of life (QoL). The description of QoL connotes

\footnotetext{
*Correspondence: toyin_akinodanye@yahoo.co.uk

${ }^{1}$ Department of Clinical Psychology, University College Hospital, Ibadan, Nigeria

Full list of author information is available at the end of the article
}

an individual's view of his/her life within the context of his/her culture, value systems, personal goals, standards and concerns [2]. Most patients being treated for $\mathrm{CaP}$ encounter undesirable challenges capable of influencing their QoL [3]. Studies show that most cancer patients are living with at least one additional comorbid illness $[4,5]$, the presence of which is associated with poorer quality of life, due to increased symptom and socioeconomic burden [6]. Cancer imposes financial burdens on a considerable percentage of patients [7, 8]. Financial toxicity describes the financial impact of cancer treatment on a 
patient's life $[9,10]$. The clinical relevance of financial distress stands out as equivalent to physical and psychological distress. In fact, financial distress can affect multiple facets of life and ultimately QoL [11]. Cancer patients with higher levels of perceived financial hardship have been shown to experience worse overall QoL [12].

Also, illness perceptions seem one of the key psychological concepts that is related to various illness outcomes. Illness perceptions are the cognitive and emotional responses of patients to their illness and its medical management [13]. These perceptions do not necessarily mirror "medically correct facts" and could be at great variance from established medical wisdom. Illness perceptions are modeled based on these five dimensions: identity, consequences, timelines, control/cure and cause [14]. In line with the assumptions of the common-sense model of self-regulation of health and illness [13], the literature abounds with the findings that illness perceptions have an impact on illness outcomes, such as functional health and QoL, directly and indirectly via illness behavior [14-18]. A meta-analysis [17] reported that the perceptions of consequences of an illness and emotional representations dimensions have the strongest relationships with psychological outcomes, including QOL, across a wide range of illnesses.

Furthermore, studies have reported on the influence of demographic characteristics (age, education and marital status) and comorbid illnesses on the quality of life of men with CaP. Age has been described as the most important factor influencing health-related QoL in patients with CaP [19]. Though older men with prostate cancer have been shown to not be different significantly from their younger counterparts in quality of life following treatment [20], other studies have reported that prostate cancer patients who were diagnosed at a younger age had the better health-related quality of life specifically in relation to higher levels of physical functioning and sexual activity [21, 22]. While some studies reported that education did not have significant influence on quality of life of cancer patients [23-25], an older study among men with prostate cancer reported that patients with lower education levels had worse physical, social and role functions and experienced more side-effects [26]. Findings on the influence of marital status on quality of life of men with prostate cancer have been inconsistent with some studies attributing better quality of life to being married [25] while others do not [27, 28]. Studies on comorbidity in cancer patients have found that patients with more comorbid illnesses have poorer quality of life [6].

Studies on the quality of life in men with prostate cancer have not carried out enough in exploring the role of financial toxicity and illness perception on the QoL of these men. This is especially important with the increasing emphasis on the provision of holistic patientcentered care so as to identify and meet the supportive care needs of $\mathrm{CaP}$ patients [29].

\section{Purpose of study}

1. To examine the relationship between financial toxicity and illness perception with quality of life in men with prostate cancer.

2. To identify the factors that influence the quality of life in men with cancer of the prostate.

\section{Methods}

This study adopted a descriptive cross-sectional design. Simple random sampling was used in recruiting research participants from the Urology Clinic of the Department of Surgery in four Nigerian tertiary health facilities located in Ilorin, Zaria, Abeokuta and Ibadan. Patients with $\mathrm{CaP}$ were identified through the senior registrars on clinic days. The research participants were eligible if they were men receiving treatment for histological diagnosis of prostate cancer, gave voluntary consent to participate in the research and were not chronically ill looking. Men receiving treatments for other urological conditions except prostate cancer were excluded from the study.

\subsection{Description of data collection sites}

The University of Ilorin Teaching Hospital (UITH) is located in the ancient city of Ilorin, the capital of Kwara State in Northcentral Nigeria; it is strategically located at the geographical and cultural confluence of the North and South, but primary ethnic group of Kwara State is Yoruba, with significant Nupe, Bariba, Hausa minorities. Agriculture is the most common occupation in the state. Ahmadu Bello University Teaching Hospital (ABUTH), Zaria, is located in Northwest Nigeria. The residents are predominantly Hausa speaking. Agriculture is the mainstay of the state's economy with over $80 \%$ of its people actively engaged in farming. Animal rearing and poultry farming are also common occupations among the Kaduna people. Hausa and Gbari languages are the most commonly spoken. The most popular religions are mainly Islam and Christianity. Federal Medical Centre (FMC), Abeokuta, and the University College Hospital (UCH), Ibadan, are both located in Southwest Nigeria and the inhabitants speak predominantly Yoruba language.

The facilities were chosen because they were tertiary health referral centers where men with $\mathrm{CaP}$ are diagnosed and treated. Though all the sites are tertiary health facilities, only ABUTH and UCH are among the four tertiary health institutions with radiotherapy centers in the 
country. The number of participants drawn from each site is shown in Table 1.

\subsection{Sample size}

An ideal sample size of 169 was obtained for this study using online sample size calculator by specifying a confidence interval of $95 \%$ ( $z$-score of 1.96), 5\% margin of error (0.05 error tolerance) and an annual estimate of 301 cases of $\mathrm{CaP}$ reported in the four tertiary health facilities [30, 31].

Two hundred questionnaires were, however, distributed to make allowance for respondents who may decline to participate in the study or for incorrectly filled questionnaires.

\subsection{Instruments for data collection}

The following instruments were used data collection:

Demographic data form Patients information was extracted from their case notes and/or directly using a predesigned data collection form. Data collected included age in years, marital status (currently married, single, divorced/separated, widowed), educational level (no formal education, primary, secondary, tertiary),

Table 1 Respondents characteristics and how they differ in FACT-P QoL and its subscales

\begin{tabular}{|c|c|c|c|c|c|c|c|}
\hline \multirow[t]{2}{*}{ Characteristics } & \multirow[t]{2}{*}{ Frequency (\%) } & \multicolumn{6}{|c|}{ ANOVA $F$ and Mean(SD) } \\
\hline & & $\overline{Q o L}$ & $A C$ & PWB & SWB & EWB & FWB \\
\hline Age & & 2.650 & 2.040 & 0.275 & 2.791 & 0.210 & $7.840^{* *}$ \\
\hline $40-60$ years & $31(17.92 \%)$ & $87.90(21.11)$ & $26.13(5.56)$ & $15.31(5.70)$ & $18.84(5.57)$ & $14.87(4.05)$ & 12.94(8.05) \\
\hline $61-80$ years & $108(62.43 \%)$ & $90.15(21.11)$ & $24.52(6.54)$ & $15.40(5.75)$ & $20.73(5.20)$ & 14.37(3.89) & 15.13(7.15) \\
\hline Above 80 years & $34(19.65 \%)$ & $80.50(22.13)$ & $22.82(7.59)$ & $14.56(5.94)$ & 18.88(3.88) & $14.68(4.77)$ & $9.56(6.73)$ \\
\hline Total & $173(100.0 \%)$ & $87.85(21.51)$ & $24.47(6.64)$ & $15.18(5.75)$ & $20.03(5.10)$ & $14.52(4.08)$ & $13.64(7.52)$ \\
\hline Educational level & & 1.026 & 2.082 & 1.572 & $3.024^{*}$ & 0.969 & 0.269 \\
\hline No formal education & $17(9.83 \%)$ & $87.94(18.62)$ & $25.06(5.88)$ & $14.41(4.26)$ & $20.76(3.87)$ & $14.18(3.71)$ & $13.53(7.85)$ \\
\hline Primary & $22(12.72 \%)$ & $83.27(20.79)$ & $21.64(6.90)$ & $16.32(6.46)$ & $17.00(5.43)$ & 14.18(3.78) & 14.14(8.05) \\
\hline Secondary & 45 (26.01\%) & $84.62(21.06)$ & $23.84(6.93)$ & $13.73(5.34)$ & $20.49(5.21)$ & $13.87(3.85)$ & $12.69(6.86)$ \\
\hline Tertiary & $82(47.40 \%)$ & $90.32(22.36)$ & $25.37(6.31)$ & $15.66(5.82)$ & $20.40(5.08)$ & $15.06(4.28)$ & 13.83(7.93) \\
\hline Total indicated & 166 (95.96\%) & $87.60(21.47)$ & $24.43(6.59)$ & $15.10(5.68)$ & $20.01(5.15)$ & $14.53(4.05)$ & $13.53(7.61)$ \\
\hline Not indicated & $7(4.04 \%)$ & & & & & & \\
\hline Total & $173(100.0 \%)$ & & & & & & \\
\hline Treatment site & & $11.654^{* *}$ & $19.145^{* *}$ & $6.848^{* *}$ & $8.568^{* *}$ & $9.540^{* *}$ & $15.076^{* *}$ \\
\hline Ibadan & 34 (19.65\%) & $95.15(25.93)$ & $27.59(5.51)$ & $18.32(6.49)$ & $18.32(5.63)$ & 14.59(3.96) & 16.32(8.49) \\
\hline Zaria & 38 (21.97\%) & $71.47(20.11)$ & $18.55(7.27)$ & $12.47(6.04)$ & $17.55(5.20)$ & $11.79(4.24)$ & $11.11(6.76)$ \\
\hline Ilorin & $59(34.10 \%)$ & $90.20(8.96)$ & $26.58(2.61)$ & $15.29(3.00)$ & 21.98(3.31) & $15.98(1.86)$ & $10.37(5.02)$ \\
\hline Abeokuta & 42 (24.28\%) & $93.45(24.27)$ & $24.36(7.50)$ & $14.95(6.65)$ & $20.90(5.40)$ & $14.88(5.11)$ & 18.36(7.23) \\
\hline Total & $173(100.0 \%)$ & $87.85(21.51)$ & $24.47(6.64)$ & $15.18(5.75)$ & $20.03(5.10)$ & $14.52(4.08)$ & $13.64(7.52)$ \\
\hline Marital status & & 1.803 & $5.463^{*}$ & 1.266 & $8.770^{* *}$ & $6.186^{* *}$ & 1.319 \\
\hline Currently Married & $130(75.14 \%)$ & $87.43(23.76)$ & $23.45(7.24)$ & 15.38(6.19) & $20.02(4.96)$ & $14.49(4.36)$ & 14.09(8.09) \\
\hline Single & $11(6.36 \%)$ & $77.27(14.21)$ & $26.00(2.72)$ & $12.73(5.46)$ & $14.73(5.75)$ & $10.91(2.88)$ & $12.91(5.50)$ \\
\hline Divorce/Separated & $29(16.76 \%)$ & $91.66(7.59)$ & $27.66(1.59)$ & $14.52(2.56)$ & $22.00(4.30)$ & $15.86(1.94)$ & $11.62(5.27)$ \\
\hline Total indicated & 170 (98.26\%) & $87.49(21.50)$ & $24.33(6.61)$ & $15.06(5.70)$ & $20.01(5.12)$ & $14.49(4.10)$ & $13.59(7.57)$ \\
\hline Not indicated & $3(1.74 \%)$ & & & & & & \\
\hline Total & $173(100 \%)$ & & & & & & \\
\hline Number of comorbid & & $10.374^{* *}$ & $22.186^{* *}$ & $3.169^{*}$ & $2.732^{*}$ & $5.813^{* *}$ & $7.220^{* *}$ \\
\hline No comorbid disease & $34(19.65 \%)$ & $93.38(22.09)$ & $26.03(6.48)$ & $16.32(6.71)$ & $18.50(5.37)$ & $15.47(4.53)$ & $17.06(7.68)$ \\
\hline 1 comorbid disease & $92(53.18 \%)$ & $90.77(17.69)$ & $26.33(4.53)$ & $15.63(4.55)$ & 20.75(4.96) & 15.04(3.38) & 13.02(7.16) \\
\hline 2 comorbid diseases & 24 (13.87\%) & $89.29(22.97)$ & $23.46(6.98)$ & $14.96(7.17)$ & $20.96(4.50)$ & $14.04(4.56)$ & 15.88(7.11) \\
\hline$>2$ comorbid diseases & $23(13.30 \%)$ & $66.48(21.86)$ & 15.83(6.96) & $11.96(6.18)$ & $18.43(5.24)$ & $11.52(4.27)$ & $8.74(6.23)$ \\
\hline Total & $173(100 \%)$ & $87.85(21.51)$ & $24.47(6.64)$ & $15.18(5.75)$ & $20.03(5.10)$ & $14.52(4.08)$ & 13.64(7.52) \\
\hline
\end{tabular}

**ANOVA F Sig at 0.01; *ANOVA F Sig at 0.05; QoL—overall quality of life (sum of all subscales); AC—prostate cancer-specific concerns; PWB—physical wellbeing; SWB-social wellbeing; EWB-emotional wellbeing; FWB_functional wellbeing 
ethnicity (Yoruba, Igbo, Hausa, others) and number of other illnesses for which they were receiving treatment.

Quality of life (QoL) The validated 39-item Functional Assessment of Cancer Therapy-Prostate (FACTP) instrument [32] was used to assess QoL in the study participants. The tool comprises of five subscales assessing physical wellbeing, social wellbeing, emotional wellbeing, functional wellbeing and prostate cancer-specific concerns. The FACT-P is scored by adding the scores of all the subscales together to yield a comprehensive health-related QoL score. All items are scored on a scale of 0 (not at all) to 4 (very much). All negatively worded items were reverse-scored. Possible scores range from 0 to 156 . Higher scores indicate better health-related QoL. The reliability coefficient of each of the subscales in our locality is physical wellbeing 0.62 , social wellbeing 0.70 , emotional wellbeing 0.43 , functional wellbeing 0.85 and 0.40 for additional concerns.

Brief illness perception questionnaire (Brief IPQ) The patients were evaluated with the brief illness perception questionnaire (Brief IPQ) in terms of cognitive and emotional representations of illness [33]. The Brief IPQ has nine subscales (except the causal question); all items are rated with a 10-point (1 to 10) response scale. Each subscale assesses one component of illness perception. Five of the subscales assess cognitive illness representations: consequences (Item 1), timeline (Item 2), personal control (Item 3), treatment control (Item 4) and identity (Item 5). Two of the items assess emotional representations: concern (Item 6) and emotional response (Item 8). One subscale assesses illness understanding (Item 7). Assessment of the causal representation is by an openended response (item 9), which asks patients to list the three most important causal factors in their illness. However, investigating the cause of this disease was not the objective of the study, question 9 was excluded from the questionnaire. Higher scores indicate more negative perception of illness. Reliability coefficient of this questionnaire by test-retest method for each of subscales was from $\mathrm{r}=0.48$ (understanding) to $\mathrm{r}=0.70$ (consequences) [33]. The instrument, however, yielded a Cronbach Alpha of 0.62 in our locality.

Financial toxicity The comprehensive score for financial toxicity (COST-FACIT) was developed to assess financial toxicity in patients with cancer [34]. The scale is made up of 12 items scored on a 5-point scale of not at all (0) to very much (4). Some items on the scale are reverse-scored. Possible scores range from 0 to 48 . There is no cutoff score established for this instrument. All negatively worded items were reverse-scored so that higher scores on the scale represent lower financial toxicity. The authors of the scale reported a Cronbach alpha of 0.92
[35]. The scale, however, yielded a Cronbach Alpha of 0.78 in our locality.

Procedure of data collection Two hundred questionnaires were distributed to men with prostate cancer during urology clinic days by trained research assistants after informed consent had been obtained. Only 173 questionnaires were retrieved and found suitable for analysis making an $86.5 \%$ retention rate.

Data analysis Data were analyzed on IBM SPSS version 21 using descriptive statistics (frequency, percentages, mean and SD), analysis of variance (ANOVA), correlation analyses and hierarchical regression analysis. ANOVA was used to compare means in QoL across groups. Correlation analyses was used to examine the relationship between QoL with financial toxicity and illness perception variables. QoL was the outcome variable on the hierarchical regression analysis model. Variables that have been shown to possibly influence QoL like age, educational level, treatment site, marital status and number of comorbid diseases were entered into the first model, financial toxicity was included in the second model, and the illness perception variables were entered into the third model as independent variables with significant level set at 0.05 .

\section{Results}

Results are based on data collected from a sample of 173 men who were receiving treatment for prostate cancer in four tertiary health facilities in Nigeria. The average age of the participants was $71.57 \pm 11.18$. Frequency distribution and the percentage of the sample group as well as analysis (ANOVA) results are shown in Table 1.

A higher percentage of the respondents had tertiary level of education $(n=82 ; 47.40 \%)$, were currently married ( $n=130 ; 75.14 \%)$, were being treated in Ilorin $(n=59 ; 34.10 \%)$ and had one comorbid disease $(n=92$; $53.18 \%)$. Significant difference was found in the overall QoL based on where participants were receiving treatments and the number of comorbid diseases they had $(P<0.01)$. Games-Howell Post Hoc Test (data not shown, but presented in Additional files 1: Appendix) showed that patients who received treatment in Zaria indicated poorer overall QoL compared to participants from other sites $(P<0.01)$ and had significantly more additional concerns $(P<0.01)$ and poorer EWB $(P<0.05)$. Also participants with 3 or more comorbid diseases had poorer overall QoL compared to those with less than 3 comorbid diseases $(P<0.01)$ and had significantly more additional concerns as well as poorer FWB and EWB $(P<0.05)$. Though no significant difference was found in overall QoL based on age, educational status and marital status, significant differences were found in some subscales. Participants aged 
61-80 years had significantly better FWB compared to those above 80 years $(P<0.05)$. In marital status, while single participants had significantly poorer SWB compared to married or divorced/separated participants

Table 2 Correlation between financial toxicity and subscales of illness perception with overall quality of life

\begin{tabular}{llll}
\hline Variables & Mean \pm SD & Range & $\begin{array}{l}\text { Correlations } \\
\text { with QoL }\end{array}$ \\
\hline QoL (FACT-P) & $87.85 \pm 21.51$ & $0-156$ & 1 \\
Financial toxicity & $26.50 \pm 10.08$ & $0-48$ & $0.416^{* *}$ \\
IIIness perception variables & & & \\
Consequences & $5.99 \pm 3.62$ & $1-10$ & $-0.451^{* *}$ \\
Timeline & $3.59 \pm 2.97$ & $1-10$ & $-0.280^{* *}$ \\
Personal control & $4.78 \pm 3.15$ & $1-10$ & $-0.159^{*}$ \\
Treatment control & $2.29 \pm 2.71$ & $1-10$ & $-0.315^{* *}$ \\
Identity & $6.11 \pm 3.18$ & $1-10$ & $-0.394^{* *}$ \\
Concern & $6.51 \pm 3.22$ & $1-10$ & $-0.388^{* *}$ \\
Illness understanding & $4.05 \pm 3.24$ & $1-10$ & $-0.332^{* *}$ \\
Emotional response & $4.05 \pm 3.67$ & $1-10$ & $-0.471^{* *}$ \\
\hline
\end{tabular}

${ }^{* *} p<0.01{ }^{*} p<0.05$ Table 3 Hierarchical regression analysis testing influence of demographic characteristics, financial toxicity and illness perception on QoL in prostate cancer patients

\begin{tabular}{|c|c|c|c|c|c|c|c|c|c|}
\hline \multirow[b]{2}{*}{ Model variables } & \multicolumn{3}{|l|}{ Model 1} & \multicolumn{3}{|l|}{ Model 2} & \multicolumn{3}{|l|}{ Model 3} \\
\hline & b Coefficient & Beta & $t$ & $\begin{array}{l}\text { b Coef- } \\
\text { ficient }\end{array}$ & Beta & $\mathbf{t}$ & b Coefficient & Beta & $\mathrm{t}$ \\
\hline Constant & 96.812 & & $7.870^{* *}$ & 83.650 & & $7.404^{* *}$ & 124.679 & & $12.410^{* *}$ \\
\hline Age & -0.114 & -0.060 & -0.733 & -0.190 & -0.100 & -1.315 & -0.027 & -0.014 & -0.237 \\
\hline Educational level & 0.428 & 0.020 & 0.251 & -2.466 & -0.116 & -1.477 & -1.801 & -0.085 & -1.371 \\
\hline Marital status & 0.501 & 0.018 & 0.227 & 0.779 & 0.028 & 0.382 & 0.421 & 0.015 & 0.228 \\
\hline Comorbid diseases & -7.562 & -0.318 & $-4.152^{* *}$ & -6.500 & -0.274 & $-3.834^{* *}$ & -2.482 & -0.104 & -1.743 \\
\hline Treatment site & 2.276 & 0.107 & 1.362 & 1.747 & 0.082 & 1.129 & 1.018 & 0.048 & 0.739 \\
\hline Financial toxicity & & & & 0.850 & 0.392 & $5.224^{* *}$ & 0.392 & 0.181 & $2.742^{*}$ \\
\hline Consequences & & & & & & & -1.310 & -0.225 & $-3.115^{* *}$ \\
\hline Timeline & & & & & & & -0.644 & -0.088 & -1.260 \\
\hline Personal control & & & & & & & 0.193 & 0.029 & 0.441 \\
\hline Treatment control & & & & & & & -0.418 & -0.053 & -0.783 \\
\hline Identity & & & & & & & -1.817 & -0.265 & $-4.314^{* *}$ \\
\hline Concern & & & & & & & -1.425 & -0.213 & $-2.951^{* *}$ \\
\hline $\begin{array}{l}\text { Illness understand- } \\
\text { ing }\end{array}$ & & & & & & & -2.091 & -0.311 & $-4.545^{* *}$ \\
\hline \multirow[t]{2}{*}{ Emotional response } & & & & -0.702 & -0.118 & -1.523 & -0.702 & -0.118 & -1.523 \\
\hline & \multicolumn{3}{|c|}{$\begin{array}{l}\text { Model } 3 \text { Summary } \\
R=0.360 \text { R Square }=0.130 \text { Adjusted } \\
R=0.102 \text { SEE }=20.400 \\
F_{(5,154)}=4.594 p<0.00\end{array}$} & \multicolumn{3}{|c|}{$\begin{array}{l}\text { Model } 2 \text { Summary } \\
R=0.511 \text { S Square }=0.262 \text { Adjusted } \\
\quad R=0.233 \text { SEE }=18.854 \\
F_{(6,153)}=9.031 ; p<0.01\end{array}$} & \multicolumn{3}{|c|}{$\begin{array}{l}\text { Model } 3 \text { Summary } \\
R=0.769 \text { R Square }=0.591 \text { Adjusted } \\
\quad R=0.552 \text { SEE }=14.408 \\
F_{(14,145)}=14.984 ; p<0.01\end{array}$} \\
\hline
\end{tabular}

$(P<0.05)$, divorced/separated participants had significantly better EWB compared to married and single participants $(P<0.05)$. However, married participants had significantly more AC compared to separated/divorced $(P<0.05)$ participants. Though significant difference was indicated between educational levels in social wellbeing, the Post Hoc Test did not detect any difference between the groups.

Table 2 shows that participants in our study had well above average score in quality of life. Financial toxicity had significant linear relationship with quality of life $(P<0.01)$, indicating that the lower the financial toxicity, the higher the quality of life. All the illness perception variables were significantly inversely correlated with quality of life $(P<0.01)$, indicating that higher negative illness perception is related with lower QoL.

The variables in the final model jointly have significant influence on QoL $(P<0.01)$ and accounted for $59.1 \%$ of variation in quality of life in men with cancer of the prostate ( $R$ Square $=0.591, R=0.769$ ). As indicated in Table 3, regression coefficients showed that the influence was significant for financial toxicity $(P<0.05)$ and four illness perception variables: consequences, identity, concern and illness understanding $(P<0.01)$, while the other subscales did not have significant

Dependent Variable: QoL (FACT-P) ${ }^{* *} p<0.01 * p<0.05$ 
influence. Though number of comorbid diseases significantly influenced QoL in the first two models $(P<0.01)$, it lost its significance in the final model $(P>0.05)$.

\section{Discussion}

The participants in our study had above average score on the overall FACT-P quality of life. Demographic differences were found in the overall QoL of the CaP patients in our study based on where participants were receiving treatments and the number of comorbid diseases they had. Patients who received treatment in Zaria indicated poorer overall QoL compared to participants from the other sites. The likely reason for this may reside in the differences in the culture and belief system between the sites. Unlike Zaria where the people are predominantly Hausa speaking and located in Northwest Nigeria, the other three sites (Ibadan and Abeokuta in Southwest and Ilorin in Northcentral Nigeria) are predominantly Yoruba speaking, share somewhat similar culture and are of close proximity to one another. Culture has been shown to account for differences in the QoL in previous studies among respondents from different ethnicities [36-38].

Patients with $\mathrm{CaP}$ in our study having three or more comorbid diseases had poorer overall QoL compared to those with none or lesser comorbid diseases which is in line with the report of an earlier study [6]. No significant difference was seen in the overall QoL of respondents based on marital status; however, findings on the difference in QoL of patients with CaP based on marital status in the literature have not been consistent $[25,27,28]$. However, our results demonstrated that the men who were single had significantly poorer social wellbeing compared to those who were married or divorced/separated participants while those who were divorced/separated had significantly better emotional wellbeing compared to married and single participants. However, married patients had significantly more additional concerns compared to those who were separated/divorced which could explain the better emotional wellbeing reported in patients who were divorced/separated.

In the current study, the variables in the final model of the hierarchical regression analysis significantly accounted for $59.1 \%$ of the variation in the QoL in men with prostate cancer. Patients experiencing less financial toxicity were associated with better QoL which is similar to the findings of previous studies [12]. Financial toxicity is often the result of endemic poverty, high healthcare cost and out-of-pocket payment for health services due to poor coverage and underutilization of health insurance schemes in Nigeria. It is closely linked with QOL because patients with financial problems often make difficult choices in an effort to defray treatment costs which may result in bankruptcy [39] and initiate the feelings of depression and anxiety that negatively impact QOL [40].

We also found inverse relationship between negative illness perceptions and QoL akin to that reported among cancer patients [36, 41, 42]. However, the illness perception variables that significantly influenced QoL were consequences, identity, concern and illness understanding. Another study found that maintaining a sense of control and existing personal identity appeared to be an important part of illness experience [43].

\subsection{Strengths and Limitations}

This was the first multi-site study assessing the influence of financial toxicity and illness perception on the QoL of $\mathrm{CaP}$ patients in Nigerian context. However, there is a need for more multi-site studies with larger sample size covering the six geopolitical zones in Nigeria to improve our understanding of the role of cultural variations in QoL and help with the generalizability of our findings. A fundamental weakness of our study is the non-reporting of Gleason scores and clinical stage of $\mathrm{CaP}$ in our study participants due to the non-availability of these in most of the patients' medical files. Gleason scores and clinical stage of disease are considered strong predictors of outcome for men with prostate carcinoma $[44,45]$. The cross-sectional design of this study is also a limitation as it did not provide us with data on changes in the QoL of $\mathrm{CaP}$ patients over time.

\section{Conclusions}

Our study has reported that financial toxicity and illness perception have significant influence on QoL of patients with CaP. Quality of life in men with prostate cancer may be improved through mitigating the financial toxicity often involved in accessing care by evolving a comprehensive national health insurance scheme that patients can be enrolled in and through subsidizing cost of treatment for men with CaP. Also, it is important for clinicians to intentionally create opportunities to continually provide appropriate counseling and information about the illness and what to expect following cancer of the prostate diagnosis and treatment to enhance appropriate and adaptive illness perceptions.

\footnotetext{
Abbreviations

CaP: Cancer of the prostate; QoL: Quality of life; FACT-P: Functional Assessment of Cancer Therapy-Prostate; AC: Additional concerns, that is, prostate cancer-specific concerns; PWB: Physical wellbeing; SWB: Social wellbeing; EWB: Emotional wellbeing; FWB: Functional wellbeing; Brief IPQ: Brief illness perception questionnaire; UCH: University College Hospital; FMCA: Federal Medical
} 
Centre, Abeokuta; UITH: University of Ilorin Teaching Hospital; ABUTHZ: Ahmadu Bello University Teaching Hospital, Zaria; HREC: Health Research Ethics Committee; UI/EC: University of Ibadan Ethics Committee.

\section{Supplementary Information}

The online version contains supplementary material available at https://doi. org/10.1186/s12301-021-00173-7.

Additional file 1. Results of Post Hoc Analysis Not Shown in the Main Document. Narratives for Tables 1A, 1B, 1C and 1E in the additional file. 1A: Participants who were above 80 years of age had significantly poorer functional wellbeing (FWB) compared to those 61-80 years. 1B: No significant difference was found in the respondents social wellbeing (SWB) based on educational levels. 1C: Participants from Zaria had significantly poorer overall QoL compared to participants from other sites. 1E: Participants with 3 or more comorbid diseases had significantly poorer overall QoL compared to those with less than 3 or no comorbid disease.

\section{Acknowledgements}

We acknowledge Ruth Agaba and Oluwaseyi Toye for providing administrative support for the project.

\section{Authors' contributions}

EA contributed to the conception and design of the study, data collection and analysis, drafting and editing of the manuscript. CO participated in study conception and design, data collection and editing the manuscript. FS participated in study conception, data collection and editing the manuscript. LS participated in data collection and editing the manuscript. MO substantively revised the draft manuscript. FO participated in editing the manuscript. All authors have read and approved the final manuscript.

\section{Funding}

The study was funded by the US National Cancer Institute (NCI) Center for Global Health and CRDF Global (Award DAA3-18-64725-1), the University of Florida Department of Pharmacotherapy and Translational Research, the University of Florida Department of Radiation Oncology and the Prostate Cancer Transatlantic Consortium (CaPTC).

\section{Availability of data and materials}

The datasets used and/or analyzed during the current study are available from the corresponding author on reasonable request.

\section{Declarations}

\section{Ethics approval and consent to participate}

Ethical approval was obtained to conduct this study (Ethical approval numbers ABUTHZ/HREC/M07/2019; UI/EC/19/0269; FMCA/470/HREC/01/2019/09). All participants read the informed consent form or had the informed consent form read to them and signed or thumb printed on the form before participating in the study.

\section{Consent for publication \\ Not Applicable.}

\section{Competing interests}

The authors declare that they have no competing interests.

\section{Author details}

${ }^{1}$ Department of Clinical Psychology, University College Hospital, Ibadan, Nigeria. ${ }^{2}$ Prostate Cancer Transatlantic Consortium (CaPTC), Orlando, FL, USA. ${ }^{3}$ Division of Urology, Department of Surgery Federal Medical Centre, Abeokuta, Nigeria. ${ }^{4}$ Biochemistry Department, University of Ilorin/University of Ilorin Teaching Hospital, Ilorin, Nigeria. ${ }^{5}$ Department of Radiology, Ahmadu Bello University/Ahmadu Bello University Teaching Hospital, Zaria, Nigeria. ${ }^{6}$ College of Pharmacy, The University of Oklahoma Health Sciences Center, Oklahoma City, OK, USA. ${ }^{7}$ College of Pharmacy and College of Medicine, University of Florida, Orlando, FL, USA.
Received: 27 January 2021 Accepted: 10 May 2021

Published online: 20 May 2021

\section{References}

1. Ferlay J, Soerjomataram I, Dikshit R, Eser S, Mathers C, Rebelo M et al (2015) Cancer incidence and mortality worldwide: sources, methods and major patterns in GLOBOCAN 2012. Int J Cancer 136(5):E359-386

2. Walsh P, Worthington JF (2012) Guide to surviving prostate cancer, 3rd edn. Hachette Book Group, New York

3. Skolarus TA, Holmes-Rovner M, Hawley ST, Dunn RL, Barr KL, Willard NR et al (2012) Monitoring quality of life among prostate cancer survivors: the feasibility of automated telephone assessment. Urology 80(5):1021-1026

4. Boyle DA (2006) Survivorship. Clin J Oncol Nurs 10(3):407-416. https:// doi.org/10.1188/06.CJON.407-416

5. McLean G, Gunn J, Wyke S, Guthrie B, Watt GCM, Blane DN et al (2014) The influence of socioeconomic deprivation on multimorbidity at different ages: a cross-sectional study. Br J Gen Pract 64(624):e440-447

6. Sarfati D, Koczwara B, Jackson C (2016) The impact of comorbidity on cancer and its treatment. CA Cancer J Clin 66(4):337-350

7. Sharp L, Carsin AE, Timmons A (2013) Associations between cancer related financial stress and strain and psychological wellbeing among individuals living with cancer. PsychoOncology 22(4):745-755

8. Sharp L, Timmons A (2016) Pre-diagnosis employment status and financial circumstances predict cancer-related financial stress and strain among breast and prostate cancer survivors. Support Care Cancer 24(2):699-709

9. O'Connor JM, Kircher SM, de Souza JA (2016) Financial toxicity in cancer care. J Community Support Oncol 14:101-106. https://doi.org/10.12788/ jCso.0239. PMID: 27058866.

10. Zafar SY, Peppercorn JM, Schrag D et al (2013) The financial toxicity of cancer treatment: a pilot study assessing out-of-pocket expenses and the insured cancer patient's experience. Oncologist 18:381-390

11. Delgado-Guay M, Ferrer J, Rieber AG et al (2015) Financial distress and its associations with physical and emotional symptoms and quality of life among advanced cancer patients. Oncologist 20:1092-1098

12. Lathan CS, Cronin A, Tucker-Seeley R, Zafar SY, Ayanian JZ, Schrag D (2016) Association of financial strain with symptom burden and quality of life for patients with lung or colorectal cancer. J Clin Oncol 34(15):1732-1740

13. Leventhal $H$, Brissette I, Leventhal EA (2003) The common-sense model of self-regulation of health and illness. In: Cameron LD, Leventhal $\mathrm{H}$ (eds) The self-regulation of health and illness behaviour. Routledge, New York

14. Leventhal $H$, Phillips LA, Burns E (2016) The common-sense model of self-regulation (CSM): a dynamic framework for understanding illness selfmanagement. J Behav Med 39:935-946

15. Hagger MS, Koch S, Chatzisarantis NLD, Orbell S (2017) The common sense model of self-regulation: meta-analysis and test of a process model. Psychol Bull 143:1117-1154

16. Broadbent E, Wilkes C, Koschwanez H, Weinman J, Norton S, Petrie KJ (2015) A systematic review and meta-analysis of the brief illness perception questionnaire. Psychol Health 30:1361-1385

17. Dempster M, Howell D, McCorry NK (2015) Illness perceptions and coping in physical health conditions: a meta-analysis. J Psychosom Res 79:506-513

18. Abubakari AR, Cousins R, Thomas C, Sharma D, Naderali EK (2016) Sociodemographic and clinical predictors of self-management among people with poorly controlled type 1 and type 2 diabetes: the role of illness perceptions and self-Efficacy. J Diabetes Res 2016:6708164

19. Porreca A, Noale M, Artibani W, Bassi PF, Bertoni F, Bracarda S et al (2018) Disease specific and general health-related quality of life in newly diagnosed prostate cancer patients: the ProsIT CNR study. Health Qual Life Outcomes 16(1):122

20. Kikkawa K, Iba A, Kohjimoto Y, Noda Y, Sonomura T, Hara I (2018) Impact of age on quality of life in patients with localized prostate cancer treated with high-dose rate brachytherapy combined with external beam radiotherapy. Int J Urol 25(4):366-371

21. Kurian CJ, Leader AE, Thong MS, Keith SW, Zeigler-Johnson CM (2018) Examining relationships between age at diagnosis and health-related 
quality of life outcomes in prostate cancer survivors. BMC Public Health 18(1):1060

22. Mardani A, Razi SP, Mazaheri R, Dianatinasab M, Vaismoradi M (2020) Health-related quality of life in prostate cancer survivors: implications for nursing care. Int J Caring Sci 13(2):1322-1332

23. Üstündag S, Zencirci AD (2015) Factors affecting the quality of life of cancer patients undergoing chemotherapy: A questionnaire study. Asia Pac J Oncol Nurs 2:17-25

24. Yýldýz Ý, Özgüroðlu M, Toptaş T, Turna H, Sen F, Yýldýz M (2013) Patterns of complementary and alternative medicine use among Turkish cancer patients. J Palliat Med 16:383-390

25. Kao Y-L, Tsai Y-S, Ou F-Y, Syu Y-J, Ou C-H, Yang W-H, Cheng H-L, Tzai T-S, Wang J-D (2015) Determinants of quality of life in prostate cancer patients: a single institute analysis. Urol Sci 26(4):254-258

26. Knight SJ, Latini DM, Hart SL, Sadetsky N, Kane CJ, DuChane J et al (2007) Education predicts quality of life among men with prostate cancer cared for in the department of veterans affairs. Cancer 109:1769-1776

27. Bellardita L, Rancati T, Alvisi MF, Villani D, Magnani T, Marenghi C et al (2013) Predictors of health-related quality of life and adjustment to prostate cancer during active surveillance. Eur Urol 64:30-36

28. Pereira RF, Daibs YS, Tobias-Machado M, Pompeo AC (2011) Quality of life, behavioral problems, and marital adjustment in the first year after radical prostatectomy. Clin Genitourin Cancer 9:53-58

29. Ferguson J, Aning J (2015) Prostate cancer survivorship: a nurse-led service model. Br J Nurs 24(Sup18):S14-S21

30. Jedy-Agba E, Odutola M, Oga E and Adebamowo C (eds) (2016) Cancer in Nigeria, 2009-2013. Nigerian National System of Cancer Registries.ISBN number: 978-978-955-030-2

31. Oluwole OP, Rafindadi AH, Shehu MS, Samaila MOA (2015) A ten-year study of prostate cancer specimens at Ahmadu Bello University Teaching Hospital (A.B.U.T.H), Zaria, Nigeria. Afr J Urol 21(1):15-18

32. Esper P, Mo F, Chodak G et al (1997) Measuring quality of life in men with prostate cancer using the functional assessment of cancer therapyprostate instrument. Urology 50:920-928

33. Broadbent E, Petrie KJ, Main J, Weinman J (2006) The brief illness perception questionnaire. J Psychosom Res 60(6):631-637

34. de Souza JA, Yap BJ, Hlubocky FJ et al (2014) The development of a financial toxicity patient-reported outcome in cancer: the COST measure. Cancer 120:3245-3253

35. de Souza JA, Yap BJ, Wroblewski K, Blinder V, Araujo FS, Hlubocky FJ, Nicholas LH, O'Connor JM, Brockstein B, Ratain MJ, Daugherty CK, Cella D (2017) Measuring financial toxicity as a clinically relevant patient-reported outcome: the validation of the comprehensive score for financial toxicity (COST). Cancer 123(3):476-484

36. Fischer MJ, Inoue K, Matsuda A, Kroep JR, Nagai S, Tozuka K, Momiyama M, Weijl NI, Langemeijer-Bosman D, Ramai S, Nortier J, Putter H, Yamaoka K, Kubota K, Kobayashi K, Kaptein AA (2017) Cross-cultural comparison of breast cancer patients' Quality of Life in the Netherlands and Japan. Breast Cancer Res Treat 166(2):459-471. https://doi.org/10.1007/ s10549-017-4417-z

37. Urzúa A, Miranda-Castillo C, Caqueo-Urízar A et al (2013) Do cultural values affect quality of life evaluation? Soc Indic Res 114:1295-1313. https:// doi.org/10.1007/s11205-012-0203-9

38. Zhang T, Shi W, Huang Z et al (2016) Influence of culture, residential segregation and socioeconomic development on rural elderly health-related quality of life in Guangxi. China Health Qual Life Outcomes 14:98. https:// doi.org/10.1186/s12955-016-0499-2

39. Ramsey SD, Blough DK, Kirchhoff AC et al (2013) Washington cancer patients found to be at greater risk for Bankruptcy than people without a cancer diagnosis. Health Aff 32:1143-1152

40. Fenn KM, Evans SB, McCorkle R et al (2014) Impact of financial burden of cancer on survivors' quality of life. J Oncol Pract 10:332-339

41. Richardson EM, Schuz N, Sanderson K, Scott JL, Schuz B (2016) Illness representations, coping, and illness outcomes in people with cancer: a systematic review and meta-analysis. PsychoOncology. https://doi.org/ 10.1002/pon.4213

42. Husson O, Thong MS, Mols F, Oerlemans S, Kaptein AA, van de Poll-Franse LV (2013) IIIness perceptions in cancer survivors: what is the role of information provision? Psychooncology 22(3):490-498

43. Clarke LH, Bennett E (2013) You learn to live with all the things that are wrong with you': gender and the experience of multiple chronic conditions in later life. Ageing Soc 33:342-360

44. Pierorazio PM, Walsh PC, Partin AW, Epstein Jl (2013) Prognostic G leason grade grouping: data based on the modified Gleason scoring system. BJU Int 111(5):753-760

45. Mapelli P, Picchio M (2015) Initial prostate cancer diagnosis and disease staging - the role of cholinePET-CT. Nat Rev Urol 12(9):510

\section{Publisher's Note}

Springer Nature remains neutral with regard to jurisdictional claims in published maps and institutional affiliations.

\section{Submit your manuscript to a SpringerOpen ${ }^{\circ}$ journal and benefit from:}

- Convenient online submission

- Rigorous peer review

- Open access: articles freely available online

- High visibility within the field

- Retaining the copyright to your article

Submit your next manuscript at springeropen.com 